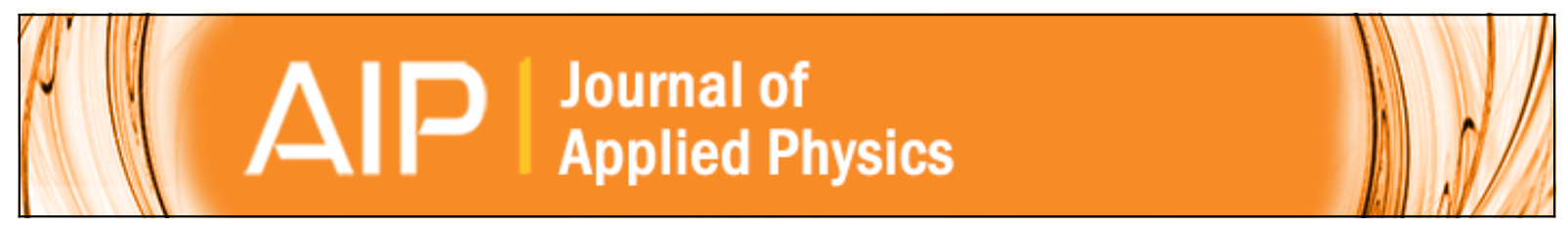

\title{
Magnetocaloric effect in Mn-containing Hitperm-type alloys
}

V. Franco, C. F. Conde, J. S. Blázquez, M. Millán, and A. Conde

Citation: Journal of Applied Physics 102, 013908 (2007); doi: 10.1063/1.2751407

View online: http://dx.doi.org/10.1063/1.2751407

View Table of Contents: http://scitation.aip.org/content/aip/journal/jap/102/1?ver=pdfcov

Published by the AIP Publishing

Articles you may be interested in

Influence of microstructure on the enhancement of soft magnetic character and the induced anisotropy of field annealed HITPERM-type alloys

J. Appl. Phys. 117, 17A301 (2015); 10.1063/1.4906173

Influence of $\mathrm{Mn}$ on the magnetocaloric effect of nanoperm-type alloys

J. Appl. Phys. 108, 073921 (2010); 10.1063/1.3489990

The magnetocaloric effect in soft magnetic amorphous alloys

J. Appl. Phys. 101, 09 C503 (2007); 10.1063/1.2709409

The influence of Co addition on the magnetocaloric effect of Nanoperm-type amorphous alloys

J. Appl. Phys. 100, 064307 (2006); 10.1063/1.2337871

Mössbauer study of FeCoNbBCu hitperm-type alloys

Appl. Phys. Lett. 81, 1612 (2002); 10.1063/1.1504164

\section{SHIMADZU Powerful, Multi-functional UV-Vis-NIR and Excellence in science FTIR Spectrophotometers}

Providing the utmost in sensitivity, accuracy and resolution for applications in materials characterization and nano research

- Photovoltaics

- Polymers

- Thin films

- Paints

- Ceramics

- DNA film structures

- Coatings

- Packaging materials
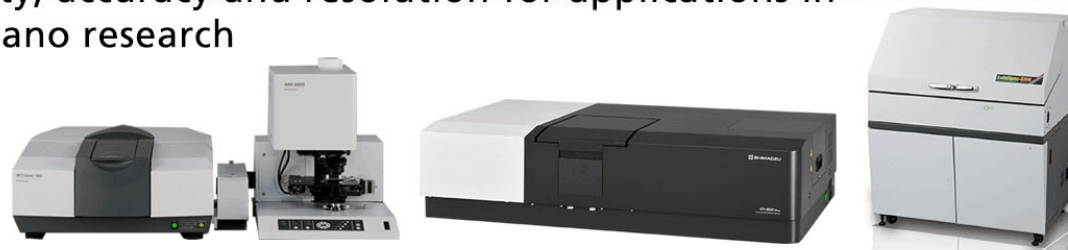


\title{
Magnetocaloric effect in Mn-containing Hitperm-type alloys
}

\author{
V. Franco, C. F. Conde, J. S. Blázquez, M. Millán, and A. Conde \\ Departimento Física de la Materia Condensada, ICMSE-CSIC, Universidad de Sevilla, P.O. Box 1065, \\ 41080 Sevilla, Spain
}

(Received 19 March 2007; accepted 16 May 2007; published online 6 July 2007)

\begin{abstract}
The magnetocaloric effect of $\mathrm{Fe}_{60-x} \mathrm{Mn}_{x} \mathrm{Co}_{18} \mathrm{Nb}_{6} \mathrm{~B}_{16}(x=0,2,4)$ is studied. Mn addition decreases the Curie temperature of the alloys but also reduces the peak entropy change and the refrigerant capacity of the material. The estimated adiabatic temperature change, for a maximum applied field of $15 \mathrm{kOe}$, is $1.3 \mathrm{~K}$. Obtained values are comparable to those of some Nanoperm-type alloys. The magnetic entropy change, $\Delta S_{M}$, of the studied samples follows a master curve, which is the same for all of them. The exponent controlling the field dependence of $\Delta S_{M}$ scales with reduced temperature in the same way as the master curve does. (C) 2007 American Institute of Physics.

[DOI: 10.1063/1.2751407]
\end{abstract}

\section{INTRODUCTION}

Magnetic refrigeration at high temperatures has become a field of increasing scientific interest since the discovery of the giant magnetocaloric effect. ${ }^{1}$ This refrigeration method, although not available yet in commercial appliances, has numerous advantages over the conventional systems based on the compression-expansion of gases: from increased energetic efficiency to environmental benefits due to the avoidance of using ozone-depleting or green-house-related gases. The main lines of research in this field have been collected in excellent reviews available in the literature. ${ }^{2-5}$ The research efforts concerning materials research can be classified in (i) the search for higher performance materials and (ii) reduction of materials cost. The first is usually centered around rare earth alloys; ${ }^{1,6,7}$ the second is being accomplished by substituting rare earth elements by transition metals. ${ }^{8}$ More recently, soft magnetic amorphous alloys are getting increasing attention as low-cost candidates for magnetic refrigeration. $^{9-18}$ (According to a well-known bibliographic database, more than $36 \%$ of the literature on the magnetocaloric effect in amorphous materials were published since 2005. Considering only the magnetocaloric effect in transition-metal-based amorphous alloys, more than $50 \%$ of the papers studying these materials were published since then.) Although the maximum magnetic entropy change, $\left|\Delta S_{M}^{p k}\right|$, for these alloys is modest when compared to that of rare-earth-based materials, ${ }^{1,6}$ the remarkable difference in material costs is an incentive for studying their suitability as magnetic refrigerants. Besides their reduced magnetic hysteresis (virtually negligible), higher electrical resistivity (which would decrease eddy current losses), tunable Curie temperature, and in the case of bulk amorphous alloys, outstanding mechanical properties, are beneficial characteristics for a successful application of the material.

Apart from their technological interest for their eventual application in refrigerators, soft magnetic amorphous materials are a good testing ground to analyze the physics behind the magnetocaloric effect, as they usually present only a $\mathrm{Cu}$ rie transition and, therefore, there is no need to consider the contribution of overlapping magnetic phenomena such as spin reorientation transition, effects of the crystalline electric field, etc. (unlike for crystalline rare earth alloys ${ }^{19,20}$ ). This simplicity allowed us to develop a master curve for the field dependence of the magnetic entropy change, ${ }^{21}$ which has been extended to lanthanide-based alloys. ${ }^{22}$ Under some circumstances (namely, alloy series with similar values of the critical exponents), this master curve can also be extended to series of alloys with different compositions, being a powerful tool for making reasonable extrapolations to field and/or temperatures outside the available experimental range in some laboratories. $^{18,23}$

Up to date, there is no published information about the magnetocaloric effect in Hitperm-type alloys, which constitute one of the well-known families of nanocrystalline alloys coming from amorphous precursors. ${ }^{24}$ Although these alloys have a high saturation magnetization, the presence of Co in the alloys causes a higher Curie temperature, $T_{C}$, of the amorphous material. However, it has been shown that $\mathrm{Mn}$ addition causes a reduction in $T_{C}$, making it closer to room temperature. Moreover, the high magnetic moment of $\mathrm{Mn}$ makes it an interesting alloying additive to analyze the magnetocaloric effect. The aim of this work is twofold: on the one hand, the performance of Mn-containing, Hitperm-type alloys will be studied, analyzing the influence of Mn addition; on the other hand, the applicability of the abovementioned master curve to this alloy series will be checked.

\section{EXPERIMENTAL}

Amorphous ribbons $(5-10 \mathrm{~mm}$ wide and $\sim 30 \mu \mathrm{m}$ thick) of $\mathrm{Fe}_{60-x} \mathrm{Mn}_{x} \mathrm{Co}_{18} \mathrm{Nb}_{6} \mathrm{~B}_{16}(x=0,2,4)$ were obtained by melt-spinning. The amorphous character of the as-quenched alloys was checked by x-ray diffraction. Details about the devitrification process of these alloys are given elsewhere. $^{25,26}$ The zero field specific heat $\left(c_{p}\right)$ of the samples (used for estimating the adiabatic temperature change, $\left.\Delta T_{a d}\right)$ has been measured in a Perkin-Elmer DSC-7 differential scanning calorimeter using $20 \mathrm{~K} / \mathrm{min}$ scans. Samples were placed in $\mathrm{Al}$ pans, and the $c_{p}$ absolute values were obtained after baseline subtraction (recorded signal of an empty Al pan). Calibration of the DSC signal was made 
using as a standard a sapphire sample in an Al pan. The field dependence of magnetization was measured in a Lakeshore 7407 vibrating sample magnetometer using a maximum applied field $H=15 \mathrm{kOe}$ with field steps of $50 \mathrm{Oe}$, for constant temperatures in the range of 300-725 K with increments of $10 \mathrm{~K}$. Prior to the measurements, the stress of the samples was relaxed by preheating them up to $700 \mathrm{~K}$.

The magnetocaloric effect, MCE, can be characterized by the magnetic entropy change due to the application of a magnetic field $H$, which can be evaluated from the processing of the temperature and field-dependent magnetization curves using a numerical approximation to the equation

$$
\Delta S_{M}=\int_{0}^{H}\left(\frac{\partial M}{\partial T}\right)_{H} d H,
$$

where the partial derivative is replaced by finite differences and the integration is performed numerically.

In order to compare the performance of different materials, either the peak entropy change, $\left|\Delta S_{M}^{p k}\right|$, or the refrigerant capacity $(R C)$ are used. The refrigeration at low temperatures requires a narrow temperature span of the refrigeration cycle, making $\left|\Delta S_{M}^{p k}\right|$ the parameter of choice for comparing low-temperature materials, while high-temperature refrigeration implies a wider temperature range and, consequently, $R C$ is employed for comparison. According to Wood and Potter, ${ }^{27}$ the $R C$ of a reversible refrigeration cycle operating between $T_{h}$ and $T_{c}$ (the temperatures of the hot and cold reservoirs, respectively) is defined as $R C=\Delta S_{M} \Delta T$, where $\Delta S_{M}$ is the magnetic entropy change at the hot and cold ends of the cycle and $\Delta T=T_{h}-T_{c}$. Moreover, hysteresis losses can be taken into account when evaluating the refrigerant material by subtracting them from the computed $R C{ }^{6}$ making the comparison between materials with different coercivities more straightforward. The optimal refrigeration cycle is that which maximizes the $R C$.

The magnetic entropy change not only depends on the measuring temperature but also on the value of the maximum applied field. The field dependence can be expressed as

$$
\Delta S_{M} \propto H^{n},
$$

where $n$ depends on the magnetic state of the sample. In order to determine the field dependence of the experimental $\left|\Delta S_{M}\right|$ data for the different magnetic regions of the studied samples, a local exponent ${ }^{9}$ can be calculated as

$$
n=\frac{d \ln \left|\Delta S_{M}\right|}{d \ln H} .
$$

\section{RESULTS AND DISCUSSION}

Figure 1 shows the isothermal magnetization curves of the 4 at. $\% \mathrm{Mn}$ alloy from which the magnetic entropy change curves have been calculated. Figure 2 shows the temperature dependence of the magnetic entropy change of the studied alloys for a maximum applied field of $15 \mathrm{kOe}$. Mn addition causes a lowering of the temperature corresponding to the peak entropy, in agreement with the previously published data which indicate a decrease in the Curie temperature of the alloy as Mn content increases. ${ }^{25}$ For temperatures

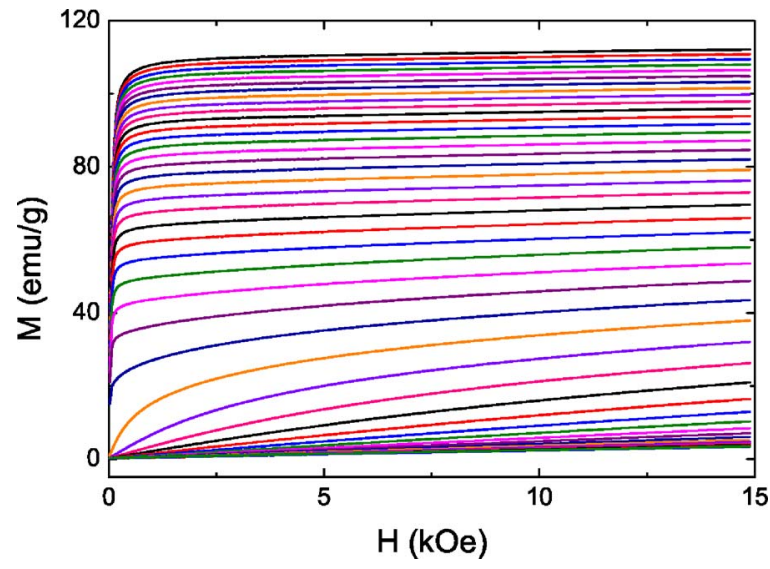

FIG. 1. (Color online) Isothermal magnetization curves for the 4 at. \% $\mathrm{Mn}$ alloy measured from $303 \mathrm{~K}$ up to $733 \mathrm{~K}$ with $10 \mathrm{~K}$ increments.

close to the higher limit of the experimental temperature range, an increase in $\Delta S_{M}$ is observed, which has a decreasing importance for alloys with increasing Mn content. This is ascribed to the beginning of the nanocrystallization process of the sample. It is known that Mn addition causes a stabilization of the amorphous alloy against nanocrystallization, ${ }^{25}$ which explains this feature of the curves. However, it has to be mentioned that the onset temperatures of the nanocrystallization process are lower than the previously published data from differential scanning calorimetry results. As the nanocrystallization process has a thermally activated character, the differences found are due to the different heating rates used for calorimetry and magnetometry experiments (the magnetization measurements of this study were made under a drastically lower effective heating rate).

In agreement with previously published data for FeZrMn amorphous alloys, Mn addition decreases the peak entropy change of the alloys. ${ }^{12}$ The refrigerant capacity also decreases with increasing Mn content, from $71 \mathrm{~J} \mathrm{~kg}^{-1} \mathrm{~K}^{-1}$ for the $\mathrm{Mn}$-free alloy to $65 \mathrm{~J} \mathrm{~kg}^{-1} \mathrm{~K}^{-1}$ for the 4 at. $\% \mathrm{Mn}$ alloy. However, the calculation of the refrigerant capacity in these alloys can be affected by the overlapping of the Curie transition originating the magnetocaloric response with the nanocrystallization of the alloys, especially for the Mn-free

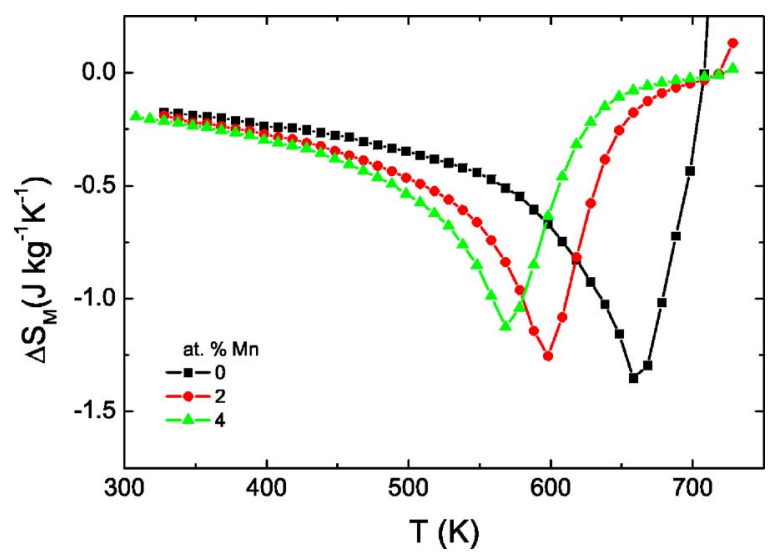

FIG. 2. (Color online) Temperature dependence of the magnetic entropy change for the studied alloys for a maximum applied field of $15 \mathrm{kOe}$. Lines are a guide for the eye. 


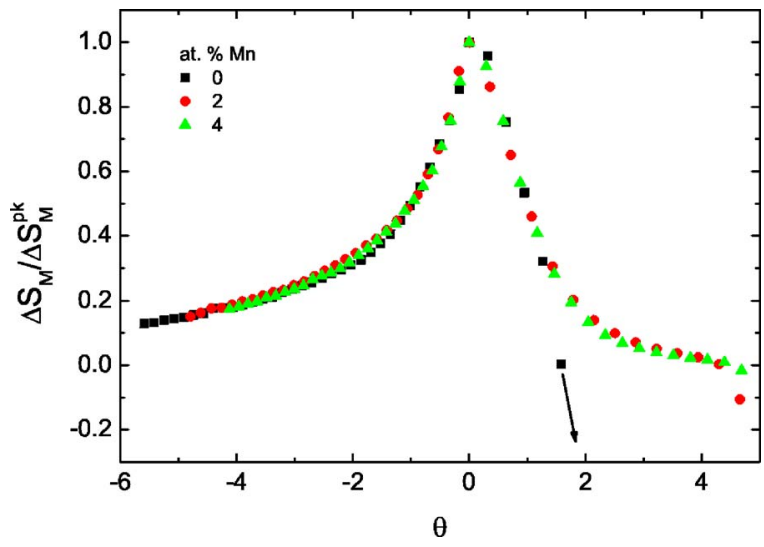

FIG. 3. (Color online) Master curve behavior for the magnetic entropy change. The arrow indicates the tendency of the data at higher reduced temperatures for the Mn-free alloy.

sample. An estimation of $\Delta T_{a d}$ can be made by using the zero field specific heat. For the Mn-free alloy, $\Delta T_{a d} \approx 1.3 \mathrm{~K}$ for a maximum applied field of $1.5 \mathrm{~T}$, decreasing to $1.1 \mathrm{~K}$ for the 4 at. $\% \mathrm{Mn}$ alloy. The values obtained for all these parameters are still comparable to those of some Nanoperm-type alloys. $^{15,23}$

It has been recently demonstrated that the $\Delta S_{M}$ curves measured with different maximum applied fields collapse into a single master curve when properly rescaled. ${ }^{21-23}$ This phenomenological master curve is obtained by plotting the normalized $\Delta S_{M}(T)$ curves versus a temperature axis which is rescaled in a different way below and above $T_{C}$. This rescaling is made by imposing that the position of two additional reference points in the curve corresponding to $\theta= \pm 1$

$$
\theta=\left\{\begin{array}{cl}
-\left(T-T_{C}\right) /\left(T_{r 1}-T_{C}\right) ; & T \leq T_{C} \\
\left(T-T_{C}\right) /\left(T_{r 2}-T_{C}\right) ; & T>T_{C}
\end{array},\right.
$$

where $T_{r 1}$ and $T_{r 2}$ are the temperatures of the two reference points which, for the present study, have been selected as those corresponding to $\frac{1}{2}\left|\Delta S_{M}^{p k}\right|$. It was also proposed ${ }^{21}$ that the $\Delta S_{M}$ curves of alloys from families of similar materials, like alloy series with minor compositional changes, should collapse into the same master curve. This proposition was subsequently confirmed with experimental results for other amorphous alloys. ${ }^{18,23}$ Figure 3 shows the master curve behavior of the magnetic entropy change curves of the studied alloys. This confirms, for this particular alloy series, the predictions of a master curve behavior for different alloy compositions of the same series. The points with $\theta>1.3$ for the Mn-free alloy, and with $\theta>4.3$ for the 2 at. $\% \mathrm{Mn}$ alloy, deviate from the master curve due to the crystallization of the sample during the measurement process. This cannot be considered a failure of the master curve, as it is only due to the change in the magnetic character of the sample, which passes from a single phase paramagnetic sample to a twophase material in which ferromagnetic nanocrystalline grains are embedded in a residual paramagnetic amorphous matrix.

The temperature dependence of the exponent $n$ controlling the field dependence of the magnetic entropy change for a maximum applied field of $15 \mathrm{kOe}$ is plotted in Fig. 4 for the studied alloys. In agreement with previous results, all

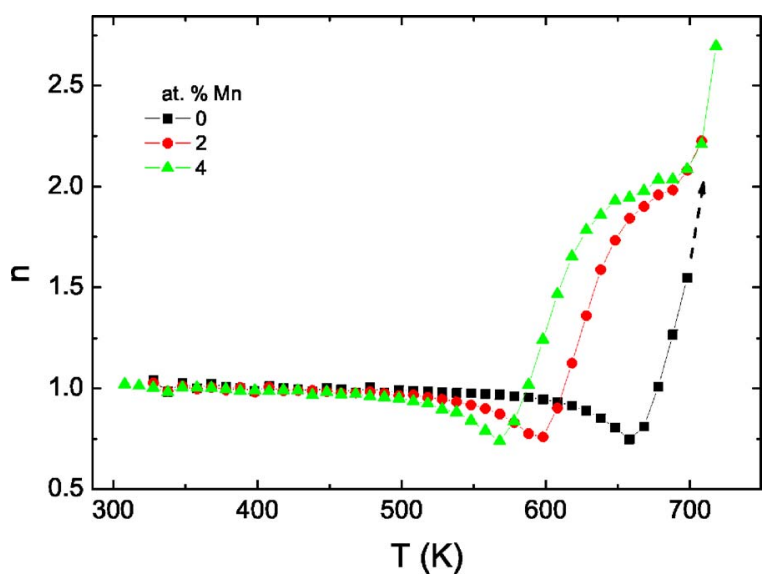

FIG. 4. (Color online) Temperature dependence of the exponent controlling the field dependence of the magnetic entropy change for a maximum applied field of $15 \mathrm{kOe}$. Lines are a guide for the eye.

samples exhibit (i) a quadratic field dependence $(n=2)$ in the paramagnetic regime (the rapidly increasing value above two for high temperatures is associated to nanocrystallization, as discussed above); (ii) a linear dependence $(n=1)$ in the ferromagnetic temperature range; and (iii) a potential law with a field exponent $n \sim 0.75$ at the Curie temperature. These values have been ascribed $^{21}$ to (i) a consequence of the linear field dependence of magnetization where the Curie-Weiss law is valid; (ii) the thermal independence of the field dependence of magnetization for moderate magnetic fields at low temperatures; and (iii) a relationship between $n$ and the two critical exponents controlling the field dependence of magnetization at the Curie temperature $(\delta)$ and the thermal dependence of magnetization $(\beta)$, of the form $n=1+(1 / \delta)[1$ $-(1 / \beta)]$, respectively.

Special attention has to be paid to the representation of $n(\theta)$ (Fig. 5), where the rescaled temperature axis $\theta$ corresponds to that previously calculated for constructing the master curve. Taking into account that the temperature dependences of the master curve and of the exponent $n$ are connected to the temperature dependence of magnetization in the different temperature regions, the collapse of these curves corroborate that the effective critical exponents of

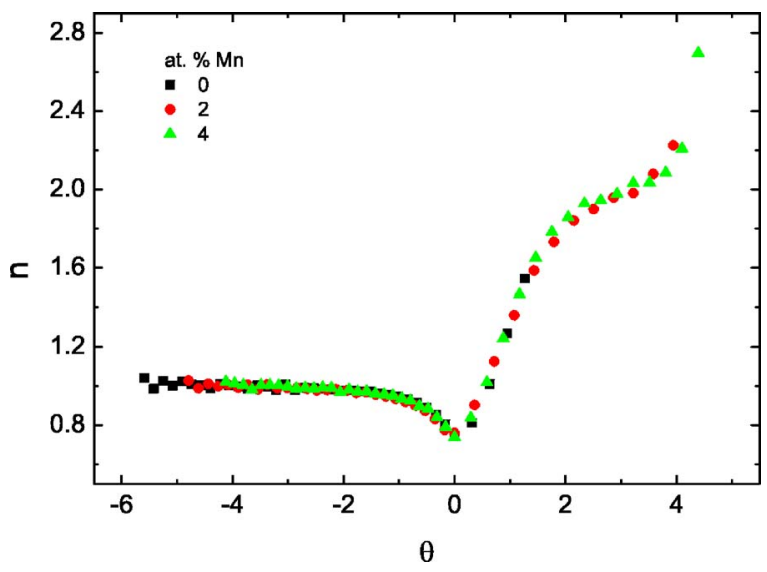

FIG. 5. (Color online) Scaling behavior of the exponent $n$ for the different studied alloys. 
these alloys should be rather similar (a requisite for constructing the master curve for collapsing data of different alloy compositions). ${ }^{21,23}$

\section{CONCLUSIONS}

The magnetocaloric response of Mn-containing Hitperm type alloys has been studied. Mn addition decreases the $\mathrm{Cu}$ rie temperature of the amorphous alloys to temperatures closer to room temperature, but also decreases the peak entropy change and the refrigerant capacity of the material. However, the values obtained for these parameters as well as for the adiabatic temperature change are still comparable to those of some Nanoperm-type alloys.

It is shown that the recently proposed extension of the master curve for the field dependence of $\Delta S_{M}$ to series of alloys with similar compositions is applicable to this particular alloy series. The exponent controlling the field dependence of the magnetic entropy change scales with reduced temperature in the same way as the $\Delta S_{M}$ curves.

\section{ACKNOWLEDGMENTS}

This work was supported by the Spanish Government and EU-FEDER (Project MAT 2004-04618) and the PAI of Junta de Andalucía (Project P06-FQM-01823). J.S.B. is grateful to Junta de Andalucía for a research contract.

${ }^{1}$ V. K. Pecharsky and K. A. Gschneidner, Jr., Phys. Rev. Lett. 78, 4494 (1997).

${ }^{2}$ A. M. Tishin, in Handbook of Magnetic Materials, edited by K. H. J. Buschow (Elsevier, Amsterdam, 1999), Vol. 12, pp. 395-524.

${ }^{3}$ K. A. Gschneidner, Jr. and V. K. Pecharsky, Annu. Rev. Mater. Sci. 30, 387 (2000)

${ }^{4}$ A. M. Tishin and Y. I. Spichkin, The Magnetocaloric Effect and Its Applications (Institute of Physics Publishing, Bristol, 2003).

${ }^{5}$ E. Brück, J. Phys. D 38, R381 (2005).
${ }^{6}$ V. Provenzano, A. J. Shapiro, and R. D. Shull, Nature 429, 853 (2004).

${ }^{7}$ T. Krenke, E. Duman, M. Acet, E. F. Wassermann, X. Moya, L. Mañosa, and A. Planes, Nat. Mater. 4, 450 (2005).

${ }^{8}$ O. Tegus, E. Bruck, K. H. J. Buschow, and F. R. de Boer, Nature 415, 150 (2002).

${ }^{9}$ T. D. Shen, R. B. Schwarz, J. Y. Coulter, and J. D. Thompson, J. Appl. Phys. 91, 5240 (2002).

${ }^{10}$ D. Wang, K. Peng, B. Gu, Z. Han, S. Tang, W. Qin, and Y. Du, J. Alloys Compd. 358, 312 (2003).

${ }^{11}$ S. Atalay, H. Gencer, and V. S. Kolat, J. Non-Cryst. Solids 351, 2373 (2005).

${ }^{12}$ S. G. Min, K. S. Kim, S. C. Yu, H. S. Suh, and S.W. Lee, J. Appl. Phys. 97, 10M310 (2005).

${ }^{13}$ V. Franco, J. S. Blázquez, C. F. Conde, and A. Conde, Appl. Phys. Lett. 88, 042505 (2006).

${ }^{14}$ F. Johnson and R. D. Shull, J. Appl. Phys. 99, 08K909 (2006).

${ }^{15}$ V. Franco, J. S. Blázquez, and A. Conde, J. Appl. Phys. 100, 064307 (2006).

${ }^{16}$ V. Franco, J. M. Borrego, A. Conde, and S. Roth, Appl. Phys. Lett. 88, 132509 (2006)

${ }^{17}$ V. Franco, J. M. Borrego, C. F. Conde, A. Conde, M. Stoica, and S. Roth, J. Appl. Phys. 100, 083903 (2006).

${ }^{18}$ V. Franco, C. F. Conde, A. Conde, and L. F. Kiss, Appl. Phys. Lett. 90, 052509 (2007).

${ }^{19}$ A. L. Lima, K. A. Gschneidner, Jr., V. K. Pecharsky, and A. O. Pecharsky, Phys. Rev. B 68, 134409 (2003).

${ }^{20}$ A. L. Lima, A. O. Tsokol, K. A. Gschneidner, Jr., V. K. Pecharsky, T. A. Lograsso, and D. L. Schlagel, Phys. Rev. B 72, 024403 (2005).

${ }^{21}$ V. Franco, J. S. Blázquez, and A. Conde, Appl. Phys. Lett. 89, 222512 (2006).

${ }^{22}$ V. Franco, A. Conde, V. K. Pecharsky, and K. A. Gschneidner, Europhys. Lett. (to be published).

${ }^{23}$ V. Franco, J. S. Blázquez, M. Millán, J. M. Borrego, C. F. Conde, and A. Conde, J. Appl. Phys. 101, 09C503 (2007).

${ }^{24}$ M. E. McHenry, M. A. Willard, and D. E. Laughlin, Prog. Mater. Sci. 44, 291 (1999).

${ }^{25}$ C.F. Conde, A. Conde, P. Svec, and P. Ochin, Mater. Sci. Eng., A 375-377, 718 (2004).

${ }^{26}$ H. Benaini, J. S. Blázquez, C. F. Conde, A. Conde, and P. Ochin, J. Alloys Compd. (in press).

${ }^{27}$ M. E. Wood and W. H. Potter, Cryogenics 25, 667 (1985). 